\title{
Disseminated Intravascular Coagulation in a Patient with Metastatic Pancreatic Neuroendocrine Tumour: A Case Report and Review of the Literature
}

\author{
Rhian Sian Davies' ${ }^{1}$ Toby Wells ${ }^{2}$, Sarah Gwynne ${ }^{1}$ \\ ${ }^{1}$ Department of Clinical Oncology, Singleton Hospital, Swansea, UK \\ ${ }^{2}$ Department of Radiology, Singleton Hospital, Swansea, UK \\ Email: rhian.s.davies@wales.nhs.uk
}

Received 27 August 2014; revised 15 September 2014; accepted 6 October 2014

Copyright (C) 2014 by authors and Scientific Research Publishing Inc.

This work is licensed under the Creative Commons Attribution International License (CC BY). http://creativecommons.org/licenses/by/4.0/

(c) (i) Open Access

\begin{abstract}
A 67-year-old female patient presented with weight loss, diarrhoea and thrombocytopaenia of unknown aetiology. A computerised tomography (CT) scan demonstrated a mass in the head of the pancreas with liver metastases. A liver biopsy demonstrated a well-differentiated neuroendocrine carcinoma. She was commenced on a somatostatin analogue. Three months later she presented with spontaneous bleeding. Blood test demonstrated results consistent with disseminated intravascular coagulation (DIC). A restaging CT scan showed evidence of disease progression. The DIC was felt to be due to the underlying progressive malignancy. Having considered the potential risks associated with cytotoxic therapy in the context of a consumptive coagulopathy, the patient was commenced on weekly Carboplatin. The patient's blood counts improved rapidly, and her bruising and bleeding resolved. Following a few weeks of stable blood results and clinical stability, her cytotoxic treatment was changed to a combination of Carboplatin and Etoposide, and to date she remains well on treatment.
\end{abstract}

\section{Keywords}

Pancreatic Neuroendocrine Tumour, Disseminated Intravascular Coagulation, Consumptive Coagulopathy

\section{Introduction}

Disseminated intravascular coagulation (DIC) is well described in patients with underlying malignancy. How-

How to cite this paper: Davies, R.S., Wells, T. and Gwynne, S. (2014) Disseminated Intravascular Coagulation in a Patient with Metastatic Pancreatic Neuroendocrine Tumour: A Case Report and Review of the Literature. Case Reports in Clinical Medicine, 3, 549-553. http://dx.doi.org/10.4236/crcm.2014.310119 
ever, no cases have previously been seen at our hospital of DIC in patients with neuroendocrine tumours. A literature search was conducted and two cases were found of patients with neuroendocrine tumours presenting with DIC. One was a patient with hepatic metastases from rectal carcinoid tumour, whose DIC resolved following resection of liver metastasis [1]. The second case was of a patient with hepatic neuroendocrine tumour metastasis from a probable pancreatic primary presenting with recurrent DIC following manipulation by core biopsy and cytotoxic therapy. The patient received one cycle of chemotherapy and subsequently deteriorated [2]. We outline the case of a patient with metastatic pancreatic neuroendocrine tumour (pNET) who presented with DIC.

\section{Case Report}

A 67-year-old female patient presented with a six months' history of gradual weight loss and diarrhoea. She had a past medical history of asthma, angioedema and urticaria, and was not taking any regular medication.

Initial blood tests demonstrated the patient was thrombocytopaenic, with a platelet count of $16 \times 10^{9} / \mathrm{L}$. Her haemoglobin was within the normal range at $116 \mathrm{~g} / \mathrm{L}$. Her coagulation results were also deranged: prothrombin time $(\mathrm{PT})>240$ seconds, activated partial thromboplastin time (APTT) $>240$ seconds, Thrombin clotting time 34.9 seconds. Her blood film was examined, and the only notable finding was thrombocytopaenia. The patient received a platelet transfusion and the thrombocytopenia improved.

A computerised tomography (CT) scan of chest, abdomen and pelvis was conducted to investigate her symptoms. This demonstrated a diffuse mass in the head of the pancreas, which obstructed the spleno-portal confluence (Figure 1 and Figure 2). Circumferential thickening of the ascending colon was also noted. Low attenuation lesions within the liver consistent with metastases were also seen (Figure 3).

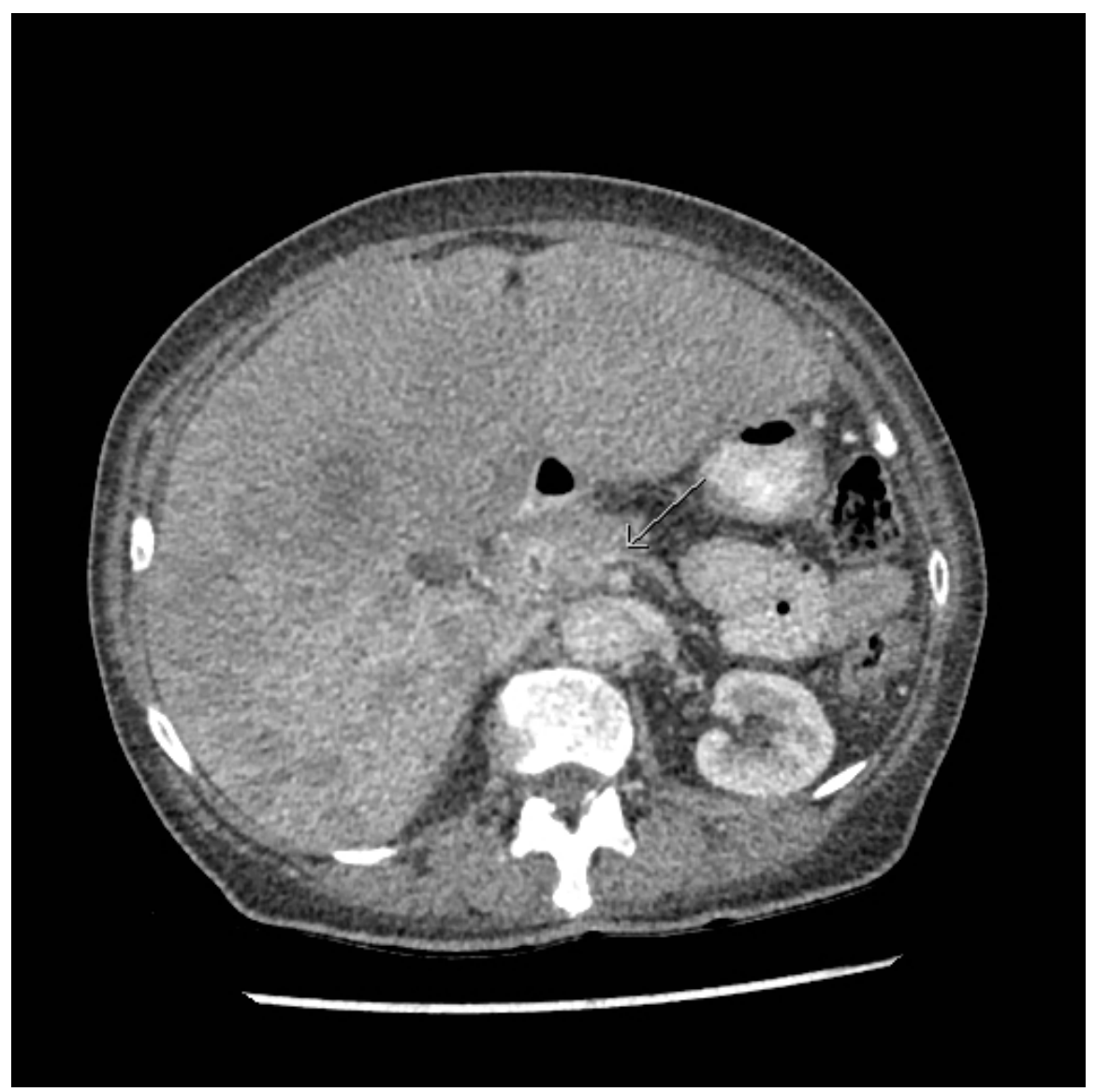

Figure 1. CT abdomen. This demonstrates attenuation of the Superior Mesenteric Vein (SMV) (arrowed) in the region of the pancreas. Below this level the SMV remains patent (Figure 2). This implies the presence of a mass in the pancreas, although the mass is ill defined and difficult to visualise. 


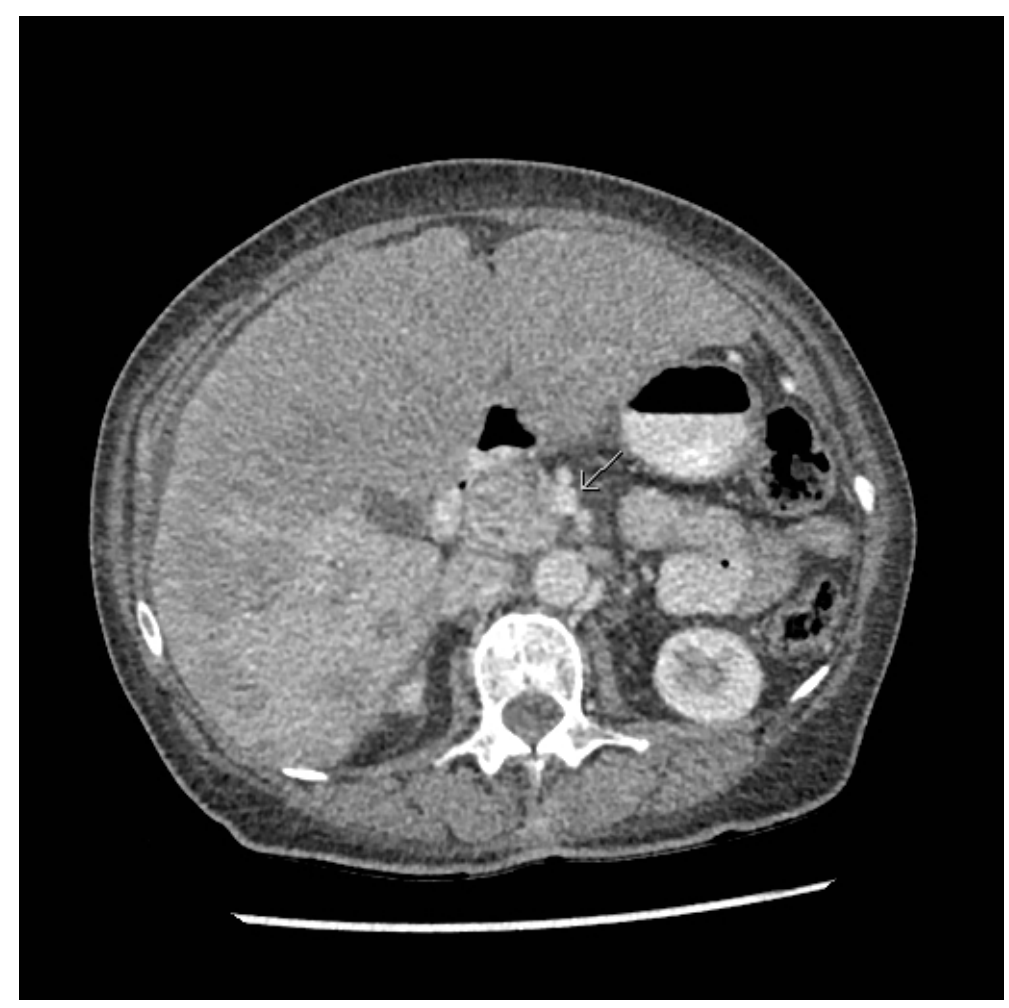

Figure 2. CT demonstrating patent SMV (arrowed) below the level of the pancreas.

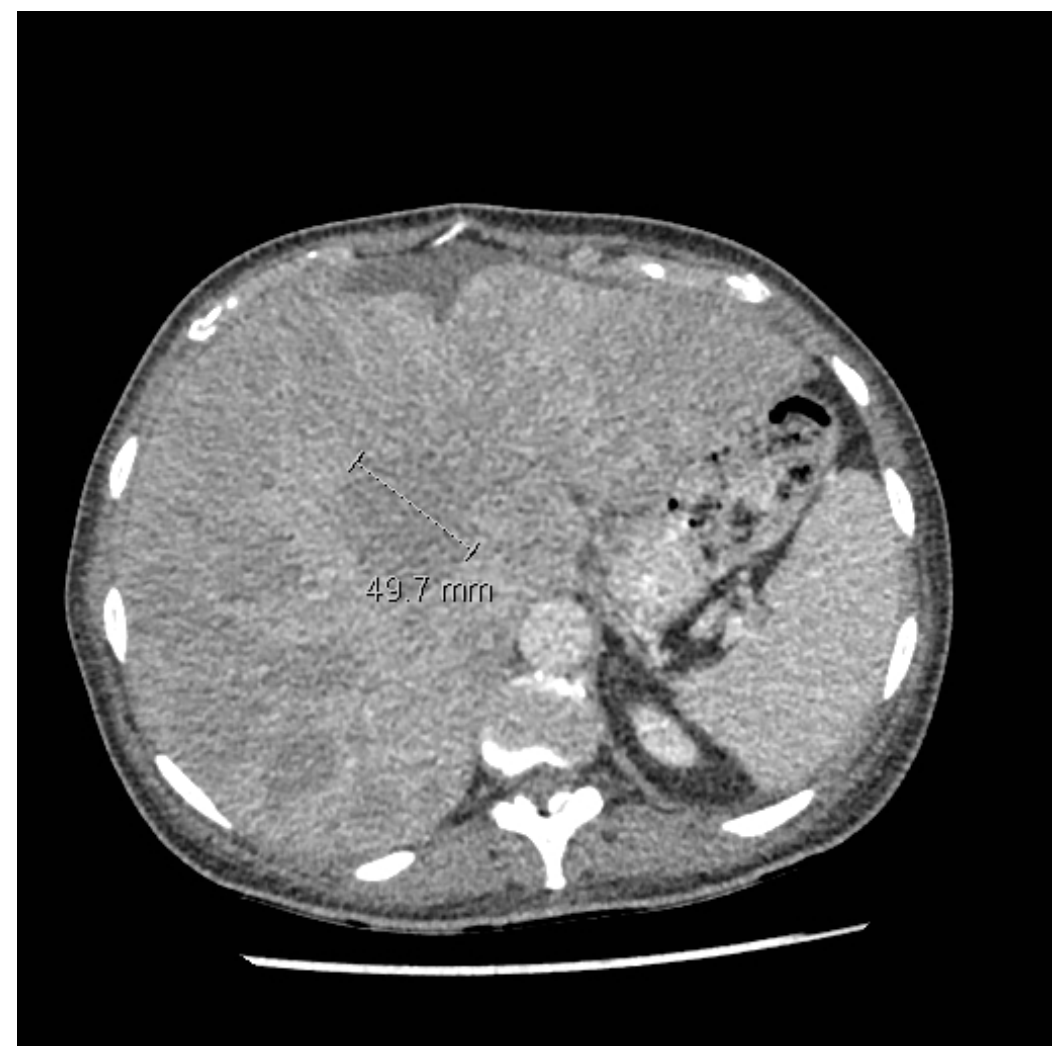

Figure 3. CT abdomen demonstrating liver metastases. 
A core biopsy of hepatic tissue was taken. This showed a malignant tumour composed of closely packed polygonal cells with both solid and glandular architecture. The cells were generally regular in appearance but with focal mild nuclear pleomorphism. There was strong positive immunochemical staining for Cam5.2, CD56, Chromogranin and Synaptohysin, and weak positivity for CK20. However, CK7, AE1/AE3, CEA, TTF1, CDX2, Ca19.9, CD117 and CK19 were negative. Mitotic figures were sparse, with no more that 1 mitosis per 10 high power ( $\times 40$ objective) microscopic fields, although the Ki67 labelling index was approximately $15 \%$. There was no evidence of tumour necrosis. It was felt that the overall appearances supported a diagnosis of metastatic, well-differentiated neuroendocrine carcinoma (predicted Grade 2). Given the CT findings, it was concluded that the patient had a metastatic neuroendocrine carcinoma of pancreatic origin.

The patient was referred to an Oncologist, and it was felt that given her metastatic unresectable disease the optimal treatment would have been combination chemotherapy. At the time of assessment the patient had a World Health Organisation (WHO) performance status of 3, and was therefore not fit enough to receive chemotherapy. There were also concerns about the use of a platinum-containing doublet given her previous thrombocytopaenia. In view of the symptoms of diarrhoea, she was commenced on Creon and a Somatostatin analogue (SomatulineAutogel $90 \mathrm{mg}$ deep subcutaneous injection 4 weekly).

Three months following commencement of somatostatin analogues the patient presented to our hospital with spontaneous bruising and bleeding from her gums. Her full blood count was checked, and she was found to have a haemoglobin level of $118 \mathrm{~g} / \mathrm{L}$ and platelet count of $23 \times 10^{9} / \mathrm{L}$. The coagulation was again deranged: PT 34.1 seconds (normal range: 9.0 - 12.5), APTT 64 seconds (normal range: 22.1 - 30.9), and fibrinogen was unrecordable. Her alkaline phosphatase was elevated at $323 \mathrm{U} / \mathrm{L}$, but otherwise her liver function and renal function tests were unremarkable. Her case was discussed with the haematology team, and the clinical picture was felt to be a consumptive coagulopathy consistent with DIC. The patient received blood products in order to stop her spontaneous bleeding.

A CT scan of her thorax, abdomen and pelvis was conducted to reassess her disease. This showed persistence of widespread liver lesions. Three index lesions had grown by about $20 \%$, which represented progressive disease. The patient also had a new left pleural effusion, but there were no obvious pulmonary deposits. Both the abnormality in the region of the pancreas and the lesion in the caecum had not changed in appearances.

In the presence of progressive disease on the CT scan the development of DIC was most likely due to the progression of her disease. There were no clinical features to suggest any other causes of DIC such as sepsis, severe liver dysfunction or trauma.

Following discussion between the specialist Oncology and Haematology teams, it was felt that the only way of treating the patient's DIC was by treating the underlying cause. The patient's symptoms had improved with the use of a somatostatin analogue, which led to an improvement in her WHO performance status. The potential for treatment with systemic chemotherapy was therefore re-considered. The main concern at this stage was worsening her coagulopathy by causing bone marrow suppression with the use of cytotoxic drugs. Following careful consideration, weekly single agent Carboplatin AUC 2 was felt to be a safer option than 3-weekly combination chemotherapy.

During the first week following the initial dose of Carboplatin, the patient experienced persistent spontaneous bruising and developed a haematoma in the lateral aspect of her left breast. She was treated with blood product support. Her blood results were monitored, and they slowly improved. By day 7 the platelet count was $54 \times$ $10^{9} / \mathrm{L}$, PT 15.4 seconds, APTT 24.5 seconds and fibrinogen was $0.5 \mathrm{~g} / \mathrm{L}$. The Carboplatin was continued on a weekly basis. By week 5, platelet count was $86 \times 10^{9} / \mathrm{L}$ and coagulation had normalised: PT 10.4 seconds, APTT 19.4 seconds and fibrinogen 1.9. The patient's condition was clinically stable, her bruising and bleeding had resolved, and her WHO performance status was now 1. Three weekly Etoposide was added to her regimen. In view of her recent problems, she received $50 \%$ of our department's standard Etoposide dose. The day 1 intravenous dose was $50 \mathrm{mg} / \mathrm{m}^{2}$, followed by $100 \mathrm{mg} / \mathrm{m}^{2}$ orally once daily on days 2 and 3 . She has tolerated the chemotherapy with no significant side effects, and remains clinically stable after 12 weeks of treatment. A repeat CT scan has demonstrated stable disease.

\section{Discussion}

\subsection{Pancreatic Neuroendocrine Tumours}

pNETs are rare. The incidence of gastro-entero-pancreatic neuroendocrine tumours is in the region of 1.1 - 5.25 
per 100,000 person years [3] [4]. pNETs form a small proportion of these cases; one case series suggests the proportion is in the region of $29 \%$ [5]. The apparent incidence has increased in recent years due to increased diagnosis via endoscopy and radiological investigations.

The heterogeneous presentation of pNETs may be attributed to the fact that $10 \%-30 \%$ of tumours are functioning whereas $70 \%-90 \%$ are non-functioning [6]. In functioning tumours, the release of peptides gives rise to classical hormonal syndromes such as Carcinoid syndrome. The majority of tumours present late with unresectable disease, mass effects and metastases [3] [6]. The 5-year survival of patients with metastatic disease is in the region of $30 \%-40 \%$ [6].

The management of pNETs depends on stage of disease, histological features and the patient's symptoms. A surgical approach is recommended for early or locoregional disease where possible. For more advanced disease cytoreductive surgery or ablative procedures (e.g. radiofrequency ablation) may be considered [4]. For functioning pNETs somatostatin analogues are used. There is evidence for the use of other therapies such as the mTOR inhibitor Everolimus, and tyrosine kinase inhibitors Sunitinib and Pazopanib in pNET. In metastatic grade 2/3 disease, chemotherapy is the treatment of choice. Combinations of cytotoxics such as Streptozocin, 5-Fluorouracil, Capecitabine and Temozolamide have been used. In the case of liver metastases (as described in this case) Etoposide and Cisplatin is the combination of choice [4] [6].

\subsection{Disseminated Intravascular Coagulation}

DIC is a syndrome characterised by activation of coagulation pathways and generation of fibrin clots. This leads to consumption of platelets and coagulation factors, which may result in bleeding. DIC does not occur in isolation, and may arise in a spectrum of disorders. These include sepsis, trauma, liver disease, vascular anomalies and malignancy [7]. Diagnosis relies on clinical suspicion and interpretation of a number of blood tests. The key to treating DIC is treatment of the underlying disorder. Supportive measures such as transfusion of platelets and plasma components may be required in patients with active bleeding [7].

\subsection{Disseminated Intravascular Coagulation in pNET}

This case represents a rare manifestation of neuroendocrine tumours, which is poorly documented, and the first documented case of DIC secondary to pNET responding to treatment with chemotherapy. The case illustrates the principle that treatment of the underlying cause of DIC may lead to resolution of the consumptive coagulopathy.

\section{References}

[1] Ito, K., Yanagisawa, S., Okada, D., et al. (2006) A Case of Giant Liver Metastases from Rectal Carcinoid Accompanied DIC. Japanese Journal of Gastroenterological Surgery, 39, 1440-1445. http://dx.doi.org/10.5833/jjgs.39.1440

[2] Teh, R.W. and Tsoi, D.T. (2012) Acute Disseminated Intravascular Coagulation in Neuroendocrine Carcinoma. Case Reports in Oncology, 5, 524-529. http://dx.doi.org/10.1159/000338401

[3] Turaga, K.K. and Kvols, L.K. (2011) Recent Progress in the Understanding, Diagnosis, and Treatment of Gastroenteropancre-Atic Neuroendocrine Tumours. CA-A Cancer Journal for Clinicians, 61, 113-132. http://dx.doi.org/10.3322/caac.20097

[4] Oberg, K., Knigge, U., Kwekkeboom, D., et al. (2012) Neuroendocrine Gastro-Entero-Pancreatic Tumors: ESMO Clinical Practice Guidelines for Diagnosis, Treatment and Follow-Up. Annals of Oncology, 23, 124-130.

[5] Pape, U.F., Bohmig, M., Tiling, N., et al. (2004) Survival and Clinical Outcome of Patients with Neuroendocrine Tuours of the Gastroenteropancreatic Tract in a German Referral Centre. Annals of the New York Academy of Sciences, 1014, 222-233. http://dx.doi.org/10.1196/annals.1294.025

[6] Valle, J.W., Eatock, M., Clueit, B., et al. (2014) A Systematic Review of Non-Surgical Treatments for Pancreatic Neuroendo-Crinetumours. Cancer Treatment Reviews, 40, 376-389. http://dx.doi.org/10.1016/j.ctrv.2014.06.003

[7] Levi, M., Toh, C.H., Thachil, J., et al. (2009) Guidelines for the Diagnosis and Management of Disseminated Intravascular Coagulation. British Journal of Haematology, 145, 24-33.

http://dx.doi.org/10.1111/j.1365-2141.2009.07600.x 
Scientific Research Publishing (SCIRP) is one of the largest Open Access journal publishers. It is currently publishing more than 200 open access, online, peer-reviewed journals covering a wide range of academic disciplines. SCIRP serves the worldwide academic communities and contributes to the progress and application of science with its publication.

Other selected journals from SCIRP are listed as below. Submit your manuscript to us via either submit@scirp.org or Online Submission Portal.
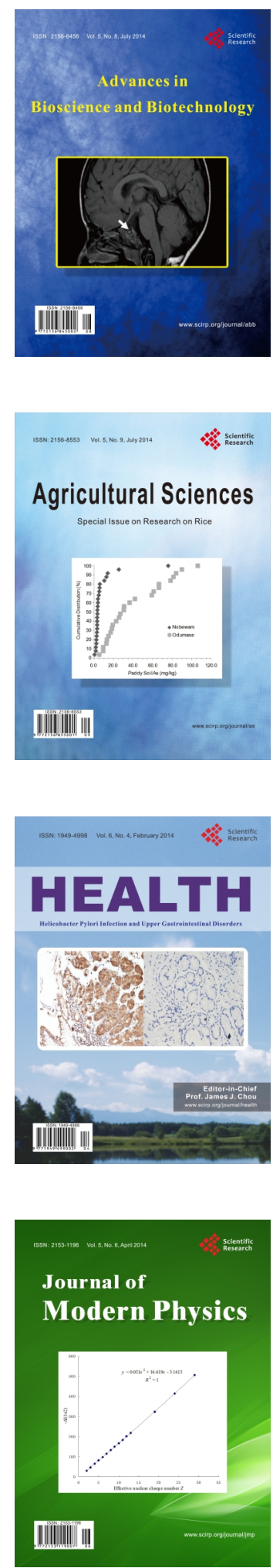
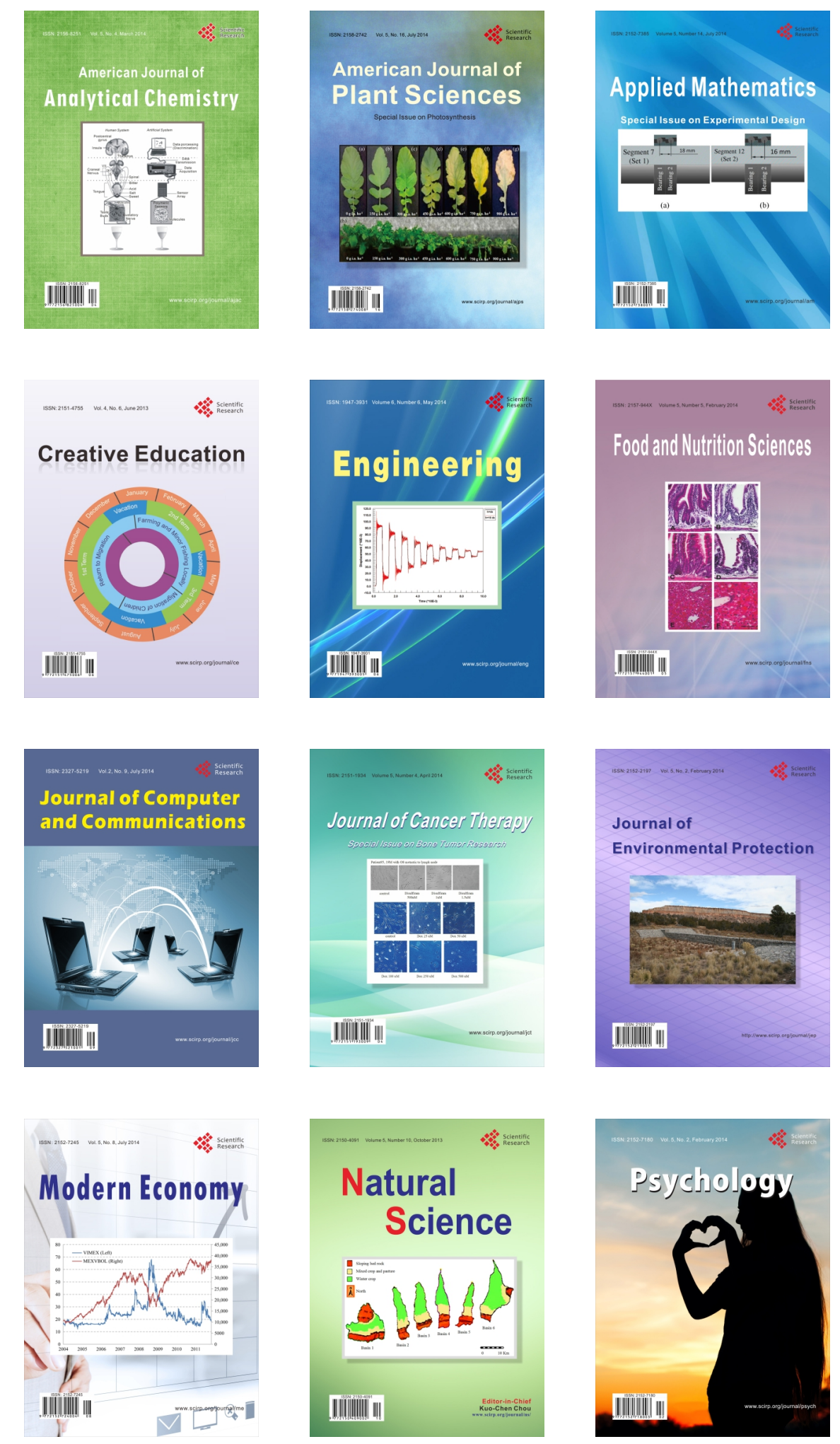